\title{
Cord blood telomere shortening associates with increased gestational age and birth weight in preterm neonates
}

\author{
NORA TABEA SIBERT ${ }^{1}$, MÓNICA S. VENTURA FERREIRA ${ }^{2}$, WOLFGANG WAGNER ${ }^{3}$, MONIKA EIPEL $^{3}$, \\ STEPHAN DRESCHERS ${ }^{1}$, TIM H. BRÜMMENDORF ${ }^{2}$, THORSTEN ORLIKOWSKY ${ }^{*}$ and FABIAN BEIER ${ }^{2 *}$ \\ ${ }^{1}$ Department of Neonatology, Medical Faculty, ${ }^{2}$ Department of Hematology, Oncology, \\ Hemostaseology and Stem Cell Transplantation, Medical Faculty, ${ }^{3}$ Helmholtz-Institute for Biomedical Engineering, \\ Stem Cell Biology and Cellular Engineering, Medical Faculty, RWTH Aachen University, D-52062 Aachen, Germany
}

Received February 9, 2020; Accepted July 21, 2020

DOI: $10.3892 /$ etm.2021.9775

\begin{abstract}
Preterm birth is considered to be associated with premature cellular aging. To address this question, two hallmarks of aging were analyzed in cord blood cells, namely telomere length and age-associated DNA methylation. Cord blood samples from 35 preterm and 11 full-term neonates were enrolled in the present study. Furthermore, quantitative telomere fluorescence in situ hybridization and flow cytometry (flow-FISH) were applied to demonstrate that telomere shortening was strongly associated with advanced gestational age and increased birth weight $\left(R^{2}=0.267\right.$ for granulocytes and $\mathrm{R}^{2}=0.307$ for lymphocytes). The estimated rate of telomere attrition in newborns during gestation ranged from 126 base pairs (bp)/week and $186 \mathrm{bp} /$ week for granulocytes and lymphocytes, respectively. In addition, neonates with longer telomeres at birth were characterized by increased weight gain during the first year of their life compared with that noted to neonates with shorter telomeres. By contrast, the epigenetic aging signature (EAS) revealed a negative correlation between epigenetic age and premature birth of unclear basis $\left(\mathrm{R}^{2}=0.26\right)$. Pending prospective validation in a larger patient cohort, the present study suggested that telomere length may be a novel biomarker alone or in combination with traditional indicators for the prediction of weight development in preterm neonates.
\end{abstract}

Correspondence to: Dr Fabian Beier, Department of Hematology, Oncology, Hemostaseology and Stem Cell Transplantation, Medical Faculty, RWTH Aachen University, 30 Pauwelsstrasse, D-52062 Aachen, Germany

E-mail: fbeier@ukaachen.de

${ }^{*}$ Contributed equally

Key words: telomere, neonates, epigenetics

\section{Introduction}

In neonatology, preterm birth is traditionally classified by gestational age in weeks and birth weight of the infant. These two markers are well-established core indicators for monitoring and evaluating perinatal health under routine health statistics $(1,2)$. However, both indicators remain descriptive and non-functional, and do not allow for functional conclusions regarding the individual biological maturity and outcome of a preterm infant. Based on the gestational age, preterm birth is divided into the following three sub-categories: i) Extremely preterm, <28 weeks; ii) very preterm, 28-32 weeks; and iii) moderate to late preterm, 32-37 weeks (1). Despite the growing knowledge on the pathophysiology of this phenomenon, preterm birth still remains a global challenge, with preterm birth rates increasing in almost all countries according to reliable data (3), thus representing the leading cause of neonatal morbidity and mortality (4). Additionally, preterm birth is also associated with long-term increased risk of adverse health outcome. Long-term effects of preterm birth include visual, hearing and neurocognitive impairment as well as an increased risk of chronic diseases such as insulin resistance (5), respiratory (6) and cardiovascular diseases (7), typical diseases of the elderly population. The aforementioned reports indicate that preterm birth may be involved in premature aging, however, further studies identifying age-associated molecular markers as potential biomarkers in preterm infants are urgently needed.

Telomeres consist of repeats of the DNA sequence TTAGGG and are located at the end of each individual chromosome arm (8). With each somatic cell division, telomere length undergoes replicative shortening as DNA polymerase is unable to fully copy telomeric DNA, the so-called end-replication problem (9). Therefore, telomeres reflect the replicative history of a cell and provide a well-established marker for organismal aging and stem cell turnover $(10,11)$. Furthermore, short telomeres have been associated with genomic instability and aging. It has been also reported that diseases characterized by disturbed telomere maintenance such as dyskeratosis congenita, are known to reflect the phenotype of premature aging (12). 
Epigenetic alterations represent an additional established hallmark of aging (13). It has been well documented that DNA methylation patterns are modified with age. Therefore, several epigenetic aging signatures (EASs) have been applied to accurately estimate chronological age in children and adults (14-17). However, only few studies have been conducted on the identification of EASs designed especially for the neonatal population and these studies exhibited limited accuracy and precision ability due to the extremes of the population distribution (18).

The aim of the present study in preterm infants was to systematically investigate telomere length at birth and apply a previously established EAS as a potential novel biomarker, alternative to gestational age and birth weight.

\section{Patients and methods}

Samples and clinical characteristics. Cord blood from 46 neonates, including 35 preterm (28-37 weeks) and 11 full-term ( $>37$ weeks) ones, was obtained immediately postpartum by midwives of the University Hospital of Aachen. All parents signed an informed consent form prior the collection of the samples and the study was approved by the ethics committee of the University Hospital of Aachen. Immediately after birth, the length, weight and head diameter of neonates were measured during routine follow-up. Detailed characteristics of the neonates are presented in Table I. Follow-up weight measurements were available for $9 / 46$ of preterm neonates.

Flow-fluorescence in situ hybridization (FISH) analysis. Telomere length was prospectively analyzed in all 46 cord blood samples. The mean telomere length of lymphocytes and granulocytes was determined using flow-FISH as previously described (19-26). Briefly, bovine thymocytes were used as an internal control and were added to the peripheral blood cells. Samples were prepared for cell denaturation and mixed with a FITC labeled (CCCTAA) 3 peptide nucleic acid (PNA) probe (Panagene Inc.) for DNA-hybridization followed by DNA counterstaining with LDS 751 (Sigma-Aldrich; Merck KGaA). Telomeric fluorescence analysis was carried out on FC-500 or Navios (both from Becton-Dickinson and Company) using forward scatter (cell volume) and LDS 751 staining for the identification of cell subsets (thymocytes, lymphocytes and granulocytes) (Fig. 1). The autofluorescence value of the respective unstained lymphocytes, granulocytes or thymocytes was subtracted from stained samples and the mean telomere length was calculated in relation to the internal control with a known telomere length. All measurements were performed single-blinded in triplicate.

DNA methylation profiles using bisulfite pyrosequencing. To determine epigenetic age, 39 cord blood samples were analyzed based on previously published EASs (14) using bisulfite pyrosequencing. This technique is used to determine the DNA methylation levels at three CG dinucleotides (CpG sites) located in ASPA, ITGA2B and PDE4C genes. Genomic DNA was isolated with the DNA Blood and Tissue kit (Qiagen). A total of $500 \mathrm{ng}$ genomic DNA were used for further experiments such as DNA bisulfite conversion and pyrosequencing. Both experiments were performed as
Table I. Clinical characteristics of the 46 analyzed neonates

\begin{tabular}{lcc}
\hline Value & $28-37 \mathrm{w}^{1} \mathrm{n}=35$ & $>37 \mathrm{w}^{2} \mathrm{n}=11$ \\
\hline Sex & & 5 \\
Female & 21 & 6 \\
Male & & \\
Birth weight & 4 & 3 \\
SGA (<10th percentile) & 30 & 8 \\
AGA (10-90th percentile) & 1 & 0 \\
LGA (>90th percentile) & & \\
Birth length (cm) & 45.5 & 49.9 \\
Average & 3.2 & 3.3 \\
SD & & \\
Birth head diameter (cm) & 31.5 & 33.8 \\
Average & 2.2 & 2.1 \\
SD & & \\
Maternal age (years) & 3 & 0 \\
$<25$ & 18 & 1 \\
$25-34$ & 12 & \\
$\geq 35$ & 2 & \\
NA & & \\
\hline
\end{tabular}

w, weeks; NA, not available; SGA, small for gestational age; AGA, appropriate for gestational age; kb, kilo base pairs; SD, standard deviation.

described previously (14). Subsequently, age prediction was performed using the pyrosequencing results obtained from an improved multivariate model that was better adjusted for cord blood samples. This model was applied on recently described pyrosequencing results form a total of 156 blood samples particularly derived from adult donors (14). Age was predicted using the following equation: Predicted age (in years) $=-0.27001 \alpha-0.30611 \beta+1.77018 \gamma+38.76516$, where $\alpha$ indicated the methylation frequency of $\operatorname{cg} 02228185$ in ASPA gene; $\beta$ the methylation frequency of $\operatorname{cg} 25809905$ in ITGA2B gene; and $\gamma$ the methylation frequency of $\mathrm{CpG}$ upstream of cg17861230 in $P D E 4 C$ gene.

Statistical analysis. Statistical analyses were performed using the GraphPad Prism v5.0 software (GraphPad Software Inc.). Subsequently, a linear regression model was applied to determine the approximate correlation between telomere length and gestational age and birth weight. $\mathrm{P}<0.05$ was considered to indicate a statistically significant difference.

\section{Results}

Telomere length. Telomere length was prospectively analyzed in 46 cord blood samples derived from 35 preterm (28-37 weeks) and 11 full-term ( $>37$ weeks) neonates. The results indicated that increasing birth weight was highly correlated with increasing gestational age (Fig. 2A: $\mathrm{R}^{2}=0.677 ; \mathrm{P}<0.001$ ). Subsequently, telomere length of granulocytes and lymphocytes from all neonates was determined by flow-FISH $(27,28)$. Telomere length was inversely correlated with gestational age in both 


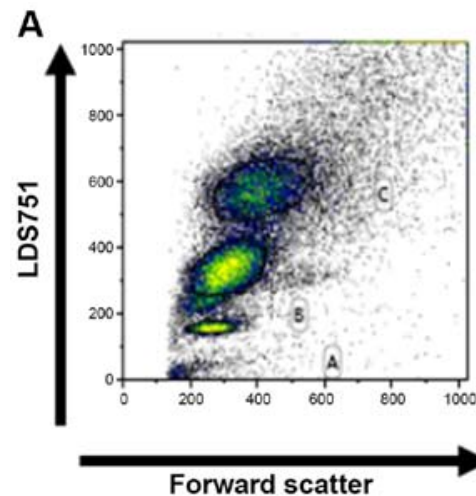

B

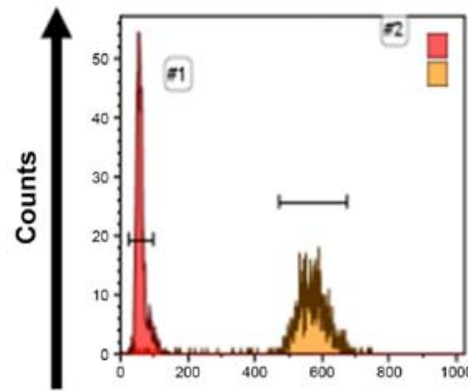

C

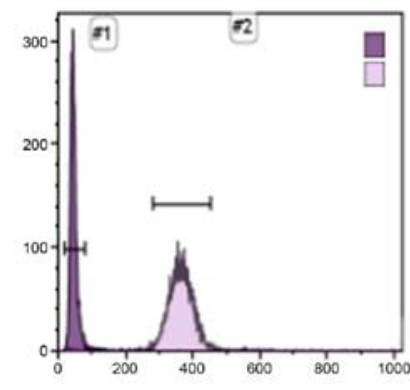

D

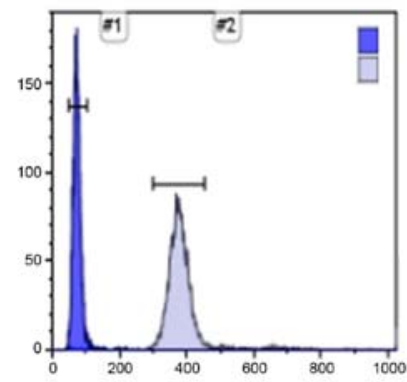

PNA-FITC (telomere signal)

Figure 1. Representative flow-FISH of the cord blood of a neonate. (A) Peripheral blood lymphocytes and granulocytes and cow thymocytes can be identified using forward scatter and DNA counterstaining with LDS751. (B) Stained and unstained cow thymocytes (C) Stained and unstained lymphocytes (D) Stained and unstained granulocytes. FISH, fluorescence in situ hybridization.

cell subpopulations (Fig. 2B and C: Granulocytes, $\mathrm{R}^{2}=0.083$; $\mathrm{P}=0.047 ; \mathrm{n}=46$; and lymphocytes, $\mathrm{R}^{2}=0.139 ; \mathrm{P}=0.011 ; \mathrm{n}=45$ ). In addition, telomere length was significantly correlated with birth weight (Fig. 2D and E: Granulocytes, $\mathrm{R}^{2}=0.267 ; \mathrm{P}<0.001$; $\mathrm{n}=46$; and lymphocytes, $\mathrm{R}^{2}=0.307 ; \mathrm{P}<0.001 ; \mathrm{n}=45$ ).

To further compare telomere shortening with known values from children and adult cohorts, the estimated telomere attrition per week and per $500 \mathrm{~g}$ weight gain was calculated using a linear regression model. Telomere shortening per week was estimated to 0.126 and $0.186 \mathrm{~kb}$ for peripheral blood granulocytes and lymphocytes, respectively. Additionally, telomere shortening per $500 \mathrm{~g}$ of weight gain was measured to 0.424 and $0.497 \mathrm{~kb}$ in the neonates' granulocytes and lymphocytes, respectively (Table II).

In the present study, data on weight gain from 9 preterm neonates born within the 32-37th week of gestational age were also available. Therefore, the postnatal weight development percentile, indicating the difference between follow-up weight percentile and initial birth weight percentile, was correlated with telomere length at the time of birth. The results revealed a positive correlation between telomere length at birth and the difference in weight development percentiles (Fig. 2F and G). More specifically, a significant correlation was observed for peripheral blood granulocytes (Fig. $1 \mathrm{~F}: \mathrm{R}^{2}=0.536 ; \mathrm{P}=0.039$; $\mathrm{n}=8$ ), but not for peripheral blood lymphocytes, where no significant trend was obtained (Fig. 2G: $\mathrm{R}^{2}=0.214 ; \mathrm{P}=0.210$; $\mathrm{n}=9$ ).

$E A S$. To further extent the biomarker analysis, a cord blood-optimized EAS was applied in 39 cord blood samples.
As expected, DNA methylation status of $P D E 4 C, I T G A 2 B$ and $A S P A$ genes at the three CpGs showed age-associated DNA methylation changes, which were consistent with previous measurements in cord blood (Fig. 3A-D). Our previously published multivariate model for age predictions was not customized for cord blood samples, therefore, the epigenetic age was systematically overestimated. To avoid bias, the multivariate model was adjusted and 39 cord blood samples were analyzed. This adjusted model provided a mean average deviation (MAD) between predicted and chronological age of 4.5 years for all samples and 2.2 years for cord blood samples. However, the application of this model requires further validation in the future using independent datasets (Fig. 3D). Furthermore, the estimated epigenetic age in the cord blood of preterm and full-term neonates was inversely correlated with gestational age (Fig. $3 \mathrm{E}: \mathrm{R}^{2}=0.26 ; \mathrm{P}=0.001 ; \mathrm{n}=39$ ). These findings were consistent with our previous study, where the aforementioned multivariate model was performed (14). By contrast, no statistically significant correlation between predicted age and birth weight was observed (Fig. 3F: $\mathrm{R}^{2}=0.07$; $\mathrm{P}=0.10 ; \mathrm{n}=39$ ).

\section{Discussion}

Preterm birth has an impact on the molecular markers of aging. The results of the present study were consistent with previously published data by Friedrich et al, demonstrating a significant correlation between birth weight and telomere length in extremely preterm infants. In the present study an accelerated rate of telomere shortening was also observed, 

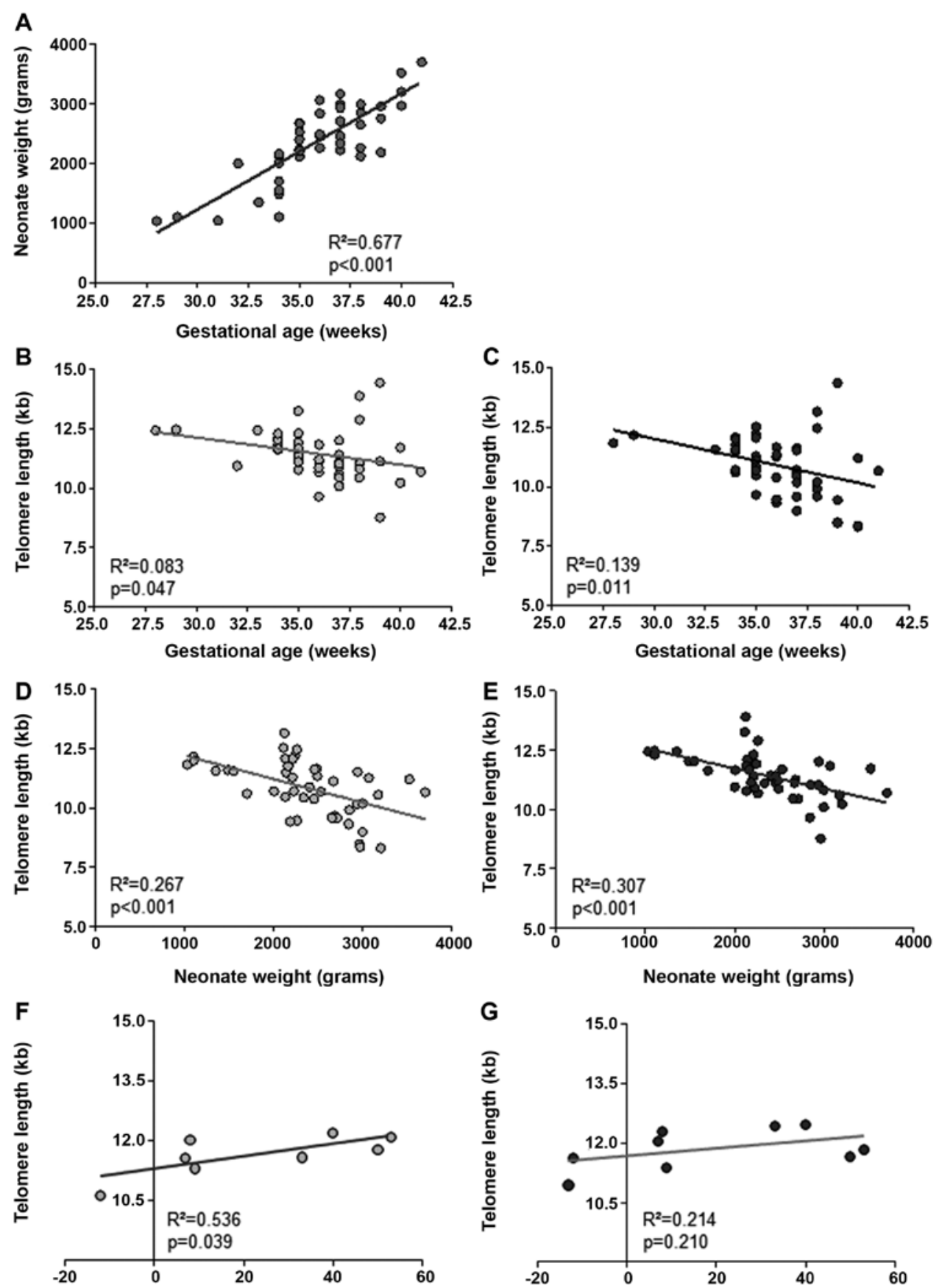

Delta (follow-up wight-birth weight) percentiles

Delta (follow-up wight-birth weight) percentiles

Figure 2. (A) Correlation between neonatal gestational age (in weeks) and birth weight (in grams). (B and C) Correlation between mean telomere length (in $\mathrm{Kb}$ ) and gestational age (in weeks) in the neonates granulocytic and lymphocytic population. Grey circles indicate (B) granulocyte and dark circles indicate (C) lymphocytic subpopulation. (D and E) Correlation between mean telomere length (in $\mathrm{Kb}$ ) and gestational age (in weeks). Grey circles indicate (D) granulocyte and dark circles indicate the (E) lymphocytic subpopulation. ( F and G) Correlation between mean telomere length (in Kb) of the neonates granulocytes and lymphocytes and the respective Delta (follow-up weight percentile-birth weight percentile). Grey circles indicate (F) granulocyte and (G) dark circles indicate lymphocytic subpopulation.

with 0.126 and $0.186 \mathrm{~kb}$ per week in granulocytes and lymphocytes, respectively. Consistent with our results, a previous study demonstrated an estimated weekly telomere shortening rate of $0.041 \mathrm{~kb}$ in leucocytes from overall preterm ( $<37$ week) infants and $0.238 \mathrm{~kb}$ in extreme to very preterm (27-32 week) born infants (29). In addition, other studies showed increased telomere shortening range during the first years of development, which was also consistent with the results of the current study $(22,30)$.
This study also suggested that telomere length was strongly correlated with birth weight, but not with gestational age. Okuda et al reported a significant correlation between telomere length in different fetal tissues and cord blood (31). Therefore, it was hypothesized that telomere length in cord blood could be considered as a more robust surrogate marker for organismal growth/maturation and weight gain compared with gestational age. The estimated telomere length shortening was approximately $0.5 \mathrm{~kb} / 500 \mathrm{~g}$ or $1 \mathrm{bp} / 1 \mathrm{~g}$ of weight gain. 
Table II. Calculated telomere shortening in the lymphocyte and granulocyte subpopulation

$\begin{array}{lrr}\text { Granulocytes } & 0.1257 \pm 0.0617 & 0.42395 \pm 0.0970 \\ \text { Lymphocytes } & 0.1862 \pm 0.0699 & 0.49680 \pm 0.1246\end{array}$
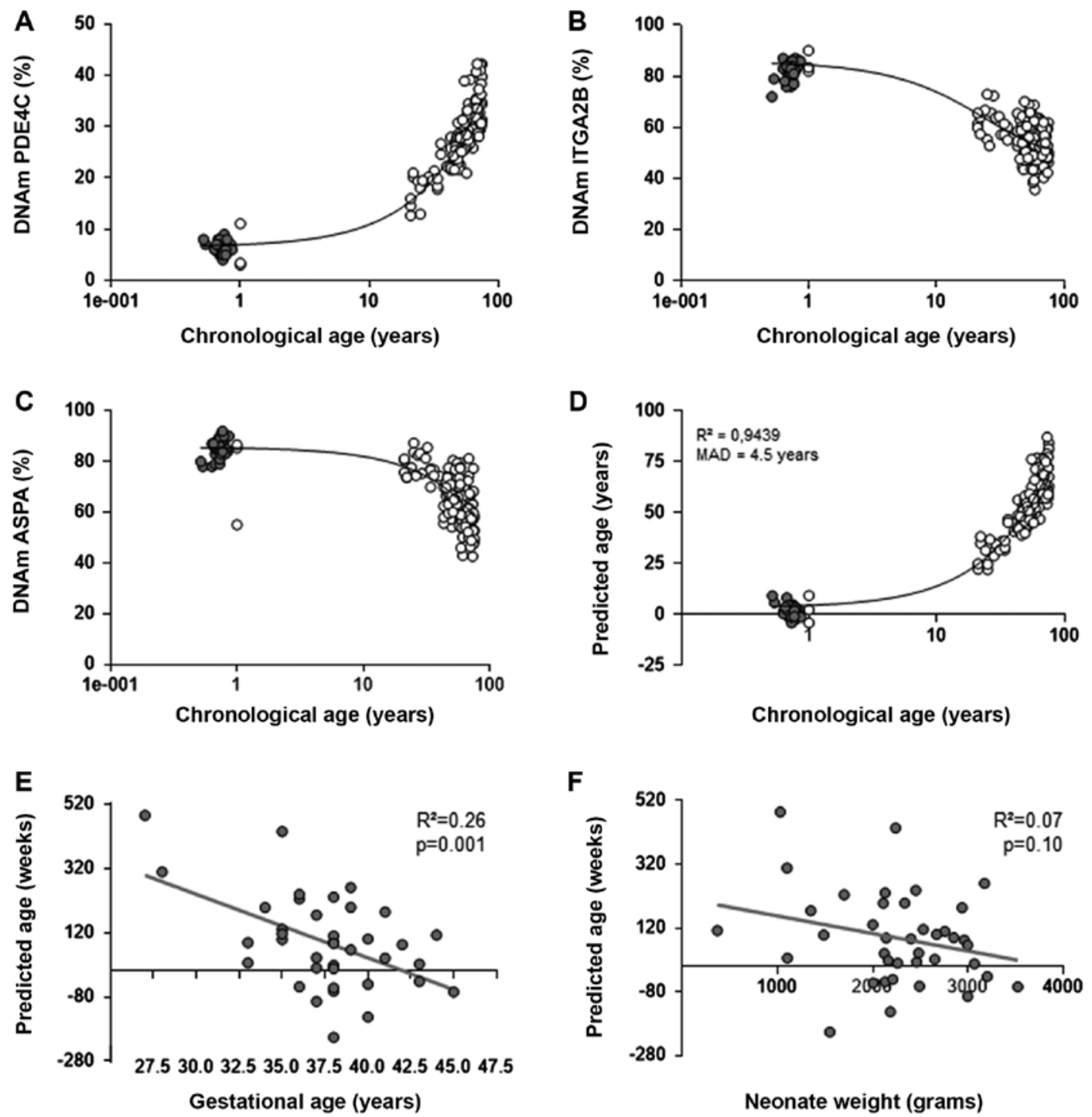

Figure 3. Correlation of gestational age and birth weight with epigenetic age (A-C) DNA methylation levels were analyzed at three age-associated CpGs associated with the genes (A) PDE4C, (B) ITGA2B and (C) ASPA in 156 blood samples of our previous work (blue) (14) and 39 cord blood samples of this study (red). The results were plotted against the logarithm of chronological age to depict age-differences of preterm cord blood. (D) Correlation of chronological age vs. predicted age based on a multivariate model with all of the above mentioned samples (MAD). The predicted ages revealed a moderate but significant inverse correlation with (E) gestational age, while there was no clear association with (F) birth weight. CpG, CG dinucleotides; MAD, mean absolute deviation.

Therefore, the longitudinally followed neonates with longer telomeres as opposed to those with shorter telomeres, reached normal weight percentiles during the first year of development. The close correlation of telomere length and weight could explain the increasing variability of telomere length with increased week of gestation. However, other factors such as food consumption or parental body mass index may influence weight gain. Therefore, it was suggested that organismal growth and weight gain could be considered as additional factors contributing to the variability of telomere length.
Regarding DNA methylation in neonates, there is limited information on epigenetic changes during gestation. The present study also revealed a significant correlation with gestational age, but not with birth weight using the optimized EAS. A previous study by Javed et al did not report any association between gestational age, birth weight and methylation profile at birth based on $353 \mathrm{CpG}$ sites and Horvath predictor for age estimation using cord blood (32). However, Knight et al established a predictor model for gestational age based on $148 \mathrm{CpG}$ sites similar to the present data (18). In general, wider aging signatures may be more 
precise, however, pyrosequencing of few $\mathrm{CpGs}$ is more cost effective and provides higher site-specific precision of the DNA methylation levels. More interestingly, the finding that epigenetic and gestational age were inversely correlated was somewhat unexpected, therefore, further validation in independent and larger cohorts is urgently needed. It has been suggested that age-related DNA methylation changes in peripheral blood occur more rapidly during childhood and are imperfectly accounted for statistical corrections that are linear in age (33). Therefore, it is conceivable that preterm birth is associated with aberrant epigenetic age and vice versa.

In summary, this study highlighted the predictive value of aging biomarkers at birth. While telomere length is correlated with the organismal growth, DNA methylation changes are correlated with maturity based on gestational age. As preterm birth still remains a great challenge for pediatricians, reliable biomarkers with high prognostic value are needed for an efficient decision-making in a clinical setting. Furthermore, the findings of the present study supported the additive use of telomere length as a possible biomarker for therapy strategy in preterm neonates, as previously proposed (34). However, the number of cases was too low to suggest any strong clinical recommendations. Therefore, further research is needed to establish telomere length as a valuable prognostic biomarker for clinicians in predicting organismal growth and development of preterm born infants. The present study reinforced not only the importance of cellular aging during fetal development, but also the critical role of telomere length in predicting newborns' health outcome (growth and development).

\section{Acknowledgements}

The authors would like to thank Lucia Vankann and Melanie Coeuru from the Department of Hematology, Oncology, Hemostaseology and Stem Cell Transplantation) for technical assistance with flow-FISH.

\section{Funding}

No funding was received.

\section{Availability of data and materials}

The datasets used and/or analyzed during the current study are available from the corresponding author on reasonable request.

\section{Author contributions}

NTS wrote the manuscript, performed experiments, collected and analyzed data; MSVF performed experiments and interpreted the data; WW and ME performed experiments and interpreted the data; SD collected and analyzed the data; THB interpreted the data and provided financial support; TO and FB designed the experiments and provided financial support; all authors reviewed the manuscript.

\section{Ethics approval and consent to participate}

All parents signed an informed consent form prior the collection of the samples and the study was approved by the ethics committee of the University Hospital of Aachen (EK 041/15).

\section{Patient consent for publication}

Not applicable.

\section{Competing interests}

The authors declare that they have no competing interests.

\section{References}

1. World Health Organization (WHO). WHO Recommendations on Interventions to Improve Preterm Birth Outcomes. World Health Organization, Geneva, 2015.

2. Santos JV, Correia C, Cabral F, Bernardes J, Costa-Pereira A and Freitas A: Should European perinatal indicators be revisited? Eur J Obstet Gynecol Reprod Biol 170: 85-89, 2013.

3. Blencowe H, Cousens S, Oestergaard MZ, Chou D, Moller AB, Narwal R, Adler A, Vera Garcia C, Rohde S, Say L and Lawn JE: National, regional, and worldwide estimates of preterm birth rates in the year 2010 with time trends since 1990 for selected countries: A systematic analysis and implications. Lancet 379: 2162-2172, 2012.

4. Kinney MV, Lawn JE, Howson CP and Belizan J: 15 Million preterm births annually: What has changed this year? Reprod Health 9: 28, 2012.

5. Salis ER, Reith DM, Wheeler BJ, Broadbent RS and Medlicott NJ: Hyperglycaemic preterm neonates exhibit insulin resistance and low insulin production. BMJ Paediatr Open 1: e000160, 2017.

6. Kwinta P and Pietrzyk JJ: Preterm birth and respiratory disease in later life. Expert Rev Respir Med 4: 593-604, 2010.

7. Demerath EW, Cameron N, Gillman MW, Towne B and Siervogel RM: Telomeres and telomerase in the fetal origins of cardiovascular disease: A review. Hum Biol 76: 127-146, 2004.

8. Greider CW and Blackburn EH: A telomeric sequence in the RNA of Tetrahymena telomerase required for telomere repeat synthesis. Nature 337: 331-337, 1989.

9. de Lange $\mathrm{T}$, How telomeres solve the end-protection problem. Science 326: 948-952, 2009.

10. Blasco MA, Telomeres and human disease: Ageing, cancer and beyond. Nat Rev Genet 6: 611-622, 2005.

11. Brümmendorf $\mathrm{TH}$ and Balabanov S: Telomere length dynamics in normal hematopoiesis and in disease states characterized by increased stem cell turnover. Leukemia 20: 1706-1716, 2006.

12. Kirwan M and Dokal I: Dyskeratosis congenita, stem cells and telomeres. Biochim Biophys Acta 1792: 371-319, 2009.

13. López-Otín C, Blasco MA, Partridge L, Serrano M and Kroemer G: The hallmarks of aging. Cell 153: 1194-1217, 2013.

14. Weidner CI, Lin Q, Koch CM, Eisele L, Beier F, Ziegler P, Bauerschlag DO, Jöckel KH, Erbel R, Mühleisen TW, et al: Aging of blood can be tracked by DNA methylation changes at just three CpG sites. Genome Biol 15: R24, 2014.

15. Horvath S: DNA methylation age of human tissues and cell types. Genome Biol 14: R115, 2013.

16. Hannum G, Guinney J, Zhao L, Zhang L, Hughes G, Sadda S, Klotzle B, Bibikova M, Fan JB, Gao Y, et al: Genome-wide methylation profiles reveal quantitative views of human aging rates. Mol Cell 49: 359-367, 2013.

17. Bocklandt S, Lin W, Sehl ME, Sánchez FJ, Sinsheimer JS, Horvath $S$ and Vilain E: Epigenetic predictor of age. PLoS One 6: e14821, 2011.

18. Knight AK, Craig JM, Theda C, Bækvad-Hansen M, Bybjerg-Grauholm J, Hansen CS, Hollegaard MV, Hougaard DM, Mortensen PB, Weinsheimer SM, et al: An epigenetic clock for gestational age at birth based on blood methylation data. Genome Biol 17: 206, 2016.

19. Beier F, Balabanov S, Buckley T, Dietz K, Hartmann U, Rojewski M, Kanz L, Schrezenmeier H and Brümmendorf TH: Accelerated telomere shortening in glycosylphosphatidylinositol (GPI)-negative compared with GPI-positive granulocytes from patients with paroxysmal nocturnal hemoglobinuria $(\mathrm{PNH})$ detected by proaerolysin flow-FISH. Blood 106: 531-533, 2005.

20. Beier F, Masouleh BK, Buesche G, Ventura Ferreira MS, Schneider RK, Ziegler P, Wilop S, Vankann L, Gattermann N, Platzbecker U, et al: Telomere dynamics in patients with del $(5 \mathrm{q})$ MDS before and under treatment with lenalidomide. Leuk Res, Sep 21, 2015 (Online ahead of print) 
21. Beier F, Foronda M, Martinez P and Blasco MA: Conditional TRF1 knockout in the hematopoietic compartment leads to bone marrow failure and recapitulates clinical features of dyskeratosis congenita. Blood 120: 2990-3000, 2012.

22. Werner B, Beier F, Hummel S, Balabanov S, Lassay L, Orlikowsky T, Dingli D, Brümmendorf TH and Traulsen A: Reconstructing the in vivo dynamics of hematopoietic stem cells from telomere length distributions. Elife 4: e08687, 2015.

23. Bartolović K, Balabanov S, Berner B, Bühring HJ, Komor M, Becker S, Hoelzer D, KanzL, Hofmann WK and BrümmendorfTH Clonal heterogeneity in growth kinetics of CD34+CD38-human cord blood cells in vitro is correlated with gene expression pattern and telomere length. Stem Cells 23: 946-957, 2005.

24. Brummendorf TH, Ersoz I, Hartmann U, Balabanov S, Wolke H, Paschka P, Lahaye T, Berner B, Bartolovic K, Kreil S, et al: Normalization of previously shortened telomere length under treatment with imatinib argues against a preexisting telomere length deficit in normal hematopoietic stem cells from patients with chronic myeloid leukemia. Ann N Y Acad Sci 996: 26-38, 2003.

25. Brümmendorf TH, Holyoake TL, Rufer N, Barnett MJ, Schulzer M, Eaves CJ, Eaves AC and Lansdorp PM: Prognostic implications of differences in telomere length between normal and malignant cells from patients with chronic myeloid leukemia measured by flow cytometry. Blood 95: 1883-1890, 2000.

26. Brümmendorf TH, Maciejewski JP, Mak J, Young NS and Lansdorp PM: Telomere length in leukocyte subpopulations of patients with aplastic anemia. Blood 97: 895-900, 2001.

27. Rufer N, Brümmendorf TH, Kolvraa S, BischoffC, Christensen K, Wadsworth L, Schulzer M and Lansdorp PM: Telomere fluorescence measurements in granulocytes and $\mathrm{T}$ lymphocyte subsets point to a high turnover of hematopoietic stem cells and memory T cells in early childhood. J Exp Med 190: 157-167, 1999.
28. Baerlocher GM, Vulto I, de Jong G and Lansdorp PM: Flow cytometry and FISH to measure the average length of telomeres (flow FISH). Nat Protoc 1: 2365-2376, 2006.

29. Friedrich U, Schwab M, Griese EU, Fritz P and Klotz U: Telomeres in neonates: New insights in fetal hematopoiesis. Pediatr Res 49: 252-256, 2001.

30. Aubert G, Baerlocher GM, Vulto I, Poon SS and Lansdorp PM: Collapse of telomere homeostasis in hematopoietic cells caused by heterozygous mutations in telomerase genes. PLoS Genet 8: e1002696, 2012.

31. Okuda K, Bardeguez A, Gardner JP, Rodriguez P, Ganesh V, Kimura M, Skurnick J, Awad G and Aviv A: Telomere length in the newborn. Pediatr Res 52: 377-381, 2002.

32. Javed R, Chen W, Lin F and Liang H: Infant's DNA methylation age at birth and epigenetic aging accelerators. Biomed Res Int 2016: 4515928, 2016.

33. Alisch RS, Barwick BG, Chopra P, Myrick LK, Satten GA, Conneely KN and Warren ST: Age-associated DNA methylation in pediatric populations. Genome Res 22: 623-632, 2012 .

34. Turner KJ, Vasu V, Greenall J and Griffin DK: Telomere length analysis and preterm infant health: The importance of assay design in the search for novel biomarkers. Biomark Med 8: 485-498, 2014 